\title{
Desmame da Ventilação Mecânica: Promova uma Estratégia*
}

\author{
Weaning from Mechanical Ventilation: Let's Perform a Strategy
}

\author{
Rosane S. Goldwasser ${ }^{1}$, Cid Marcos David ${ }^{2}$
}

\section{RESUMO}

JUSTIFICATIVA E OBJETIVOS: O desmame da ventilação mecânica ainda é um desafio na Unidade de Terapia Intensiva (UTI) e está relacionado a complicações e a mortalidade. A visita diária aos pacientes internados pela equipe de saúde faz parte das boas práticas e pode identificar aqueles capazes de serem submetidos a um teste em ventilação espontânea. O objetivo deste estudo foi sugerir uma técnica de memorização com o termo "Estratégia" considerando alguns aspectos chave que podem ser aplicados por qualquer pessoa da equipe multidisciplinar, durante a ronda à beira do leito, a fim de abreviar o desmame.

CONTEÚDO: Introduzir a palavra "Estratégia" como um método de memorização, baseado nos estudos relacionados ao desmame da ventilação mecânica e sua aplicabilidade, como um checklist em qualquer UTI pela equipe multidisciplinar, em que cada letra lembra alguns aspectos chave relacionados ao assunto.

CONCLUSÕES: A aplicação de mecanismos de me-

1. Mestre em Clínica Médica - Setor Terapia Intensiva pela UFRJ; Coordenadora da Residência Médica do Hospital Universitário Clementino Fraga Filho da UFRJ; Coordenadora da Pós-Graduação do Fundo Brasileiro de Educação e Pesquisa-Fundo AMIB; Presidente da Sociedade de Terapia Intensiva do Estado do Rio de Janeiro - Biênio 2005-2006 e 2007-2008.

2. Professor Adjunto da Faculdade de Medicina da UFRJ; Doutor em Ciências Médicas pela UFRJ; Diretor do Centro de Tratamento Intensivo do Hospital Universitário Clementino Fraga Filho da UFRJ; Presidente do Fundo Brasileiro de Educação e Pesquisa-Fundo AMIB

${ }^{*}$ Recebido do Centro de Tratamento Intensivo do Hospital Universitário Clementino Fraga Filho da Universidade Federal do Rio de Janeiro (UFRJ), Rio de Janeiro, RJ

Apresentado em 14 de junho de 2006

Aceito para publicação em 03 de janeiro de 2007

Endereço para correspondência:

Dra. Rosane Sonia Goldwasser

Rua Guilhermina Guinle 170/701

22270-060 Rio de Janeiro, RJ

Fone: (21) 2562-6013

E-mail: rosanegold@uol.com.br

(C)Associação de Medicina Intensiva Brasileira, 2007 morização como checklist para o desmame de pacientes da ventilação mecânica, pode ser facilmente praticada durante a ronda diária para identificar aqueles aptos a realizar um teste de ventilação espontânea.

Unitermos: Desmame, Estratégia, Protocolo, Segurança, Ventilação Mecânica.

\section{SUMMARY}

BACKGROUND AND OBJECTIVES: Weaning patients from mechanical ventilation is still a challenge in Intensive Care Units (ICU) and is related to complications and mortality. Daily rounds at the bedside, which are part of good care, can identify patients able to undergo to spontaneous breathing trials. The authors suggest one mnemonic technique with the term "ESTRATEGIA" (strategy) considering some key aspects, as a checklist, which can be applied by any person of the multidisciplinary team, during the bedside rounds, in order to shorten the weaning time.

CONTENTS: To introduce the word Strategy as a mnemonic method based on the studies related to weaning from mechanical ventilation and its applicability as a checklist in any intensive care unit by the multidisciplinary team where each letter reminds some key aspects related to the subject.

CONCLUSIONS: The applicability of a mnemonic mechanism as a checklist for weaning patients from mechanical ventilation easily practiced during the daily round to identify those who are able to undergo to spontaneous breathing trials.

Key Words: Mechanical Ventilation, Protocol, Safety, Strategy, Weaning

\section{INTRODUÇÃO}

Estima-se que aproximadamente $40 \%$ dos pacientes internados nas Unidades de Terapia Intensiva (UTI) encontram-se em ventilação mecânica sendo, portanto, atitude terapêutica de alta prevalência ${ }^{1,2}$. Postergar a retirada da ventilação mecânica correlaciona-se com complicações como pneumonia, barotrauma, lesões laringotraqueais, 
repercussões hemodinâmicas, tromboembolismo, atrofia muscular e toxicidade pelo oxigênio. Estas complicações variam com a gravidade da doença, o tempo de permanência em prótese ventilatória e técnicas ventilatórias ${ }^{2-7}$. A precocidade desta remoção pode, por outro lado, relacionar-se com outros riscos, como dificuldade do acesso às vias aéreas, prejuízo na troca gasosa, além do aumento de pneumonia e da mortalidade ${ }^{8-10}$.

Retirar o paciente da ventilação mecânica pode ser mais difícil que mantê-lo. Apesar disso, a literatura tem contribuído para ressaltar que o método empregado pode alterar o desfecho do desmame e o juízo clínico pode ser menos acurado do que um protocolo bem elaborado. Tanto a percepção do momento para iniciar a interrupção do suporte ventilatório, quanto à escolha da forma mais adequada de fazê-lo, pode ser orientada com base nas evidências científicas, com melhora nos resultados obtidos ${ }^{3,5}$. Vários estudos referentes ao desmame da ventilação mecânica foram realizados nas últimas duas décadas ${ }^{11-19}$. Talvez tenha sido nesta área do conhecimento, o capítulo mais bem definido em relação a protocolos e evidências científicas. No entanto, ainda se percebe a diversidade de resultados nas várias UTI do mundo, quando se estuda a retirada da ventilação mecânica ${ }^{13,20}$. Alguns autores propõem mecanismos facilitadores onde se possa, partindo das informações clínicas e laboratoriais à beira do leito, colocar em prática a capacidade de pensar, decidir e realizar o desfecho. O objetivo deste estudo foi mostrar uma proposta simples para aplicar durante a ronda à beira do leito, através de um método de memorização utilizando uma palavra empregada nas unidades militares e, atualmente adaptada para as atividades gestoras: Estratégia.

\section{ESTRATÉGIA PROPOSTA PARA O DESMAME}

Criar mecanismos facilitadores na UTI pode melhorar e agilizar o trabalho da equipe multidisciplinar. Ao se utilizar o termo ESTRATÉGIA, como método mnemônico, os profissionais da equipe multidisciplinar podem estar aptos a identificar o paciente capaz de se submeter ao teste de ventilação espontânea ou método de interrupção e com isso abreviar o tempo de ventilação mecânica (Figura 1 e Tabela 1).

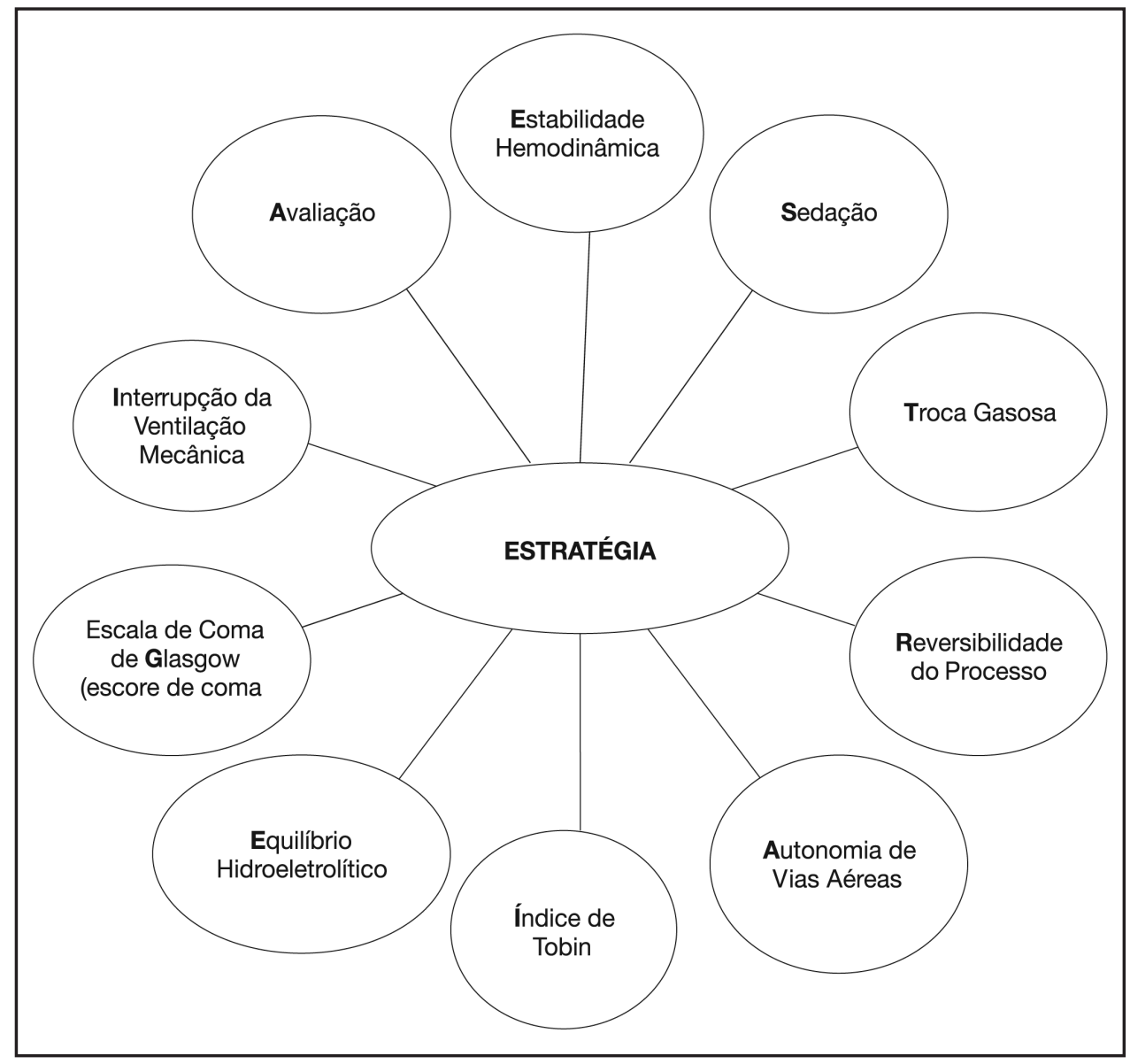

Figura 1 - Estratégia para o Desmame. 
Tabela 1 - Componentes da Estratégia

\begin{tabular}{|c|c|}
\hline Componentes & Considerações \\
\hline $\begin{array}{l}\text { Estabilidade hemodinâmi- } \\
\text { ca }\end{array}$ & $\begin{array}{l}\text { Ausência de sinais de má perfusão tecidual, insuficiência coronariana, ou dependência de vasopressores } \\
\text { por insuficiência circulatória, ausência de arritmias com repercussão hemodinâmica. }\end{array}$ \\
\hline Sedação & Ausência de agitação ou intensa sonolência, avaliados pelo grau de sedação utilizando a escala do serviço. \\
\hline Troca gasosa $2,23,24$ & $\mathrm{PaO}>60 \mathrm{mmHg}$ com $\mathrm{FiO}_{2}<0,4$ e PEEP $<5 \mathrm{cmH}_{2} \mathrm{O}$ \\
\hline Reversibilidade/controle & Da doença que causou ou contribuiu para a descompensação respiratória \\
\hline Autonomia das vias aéreas & Avaliada pela tosse eficaz, no caso de tubo traqueal ou pela presença de traqueostomia e tosse assistida. \\
\hline $\begin{array}{l}\text { Tobin ou índice de respira- } \\
\text { ção rápida e superficial } 22\end{array}$ & Razão entre a freqüência respiratória e o volume-corrente $<105$ irpm/L. \\
\hline $\begin{array}{l}\text { Equilíbrio hidroeletrolítico e } \\
\text { ácido-básico. }\end{array}$ & $\begin{array}{l}\text { Avaliação clínica ou por métodos invasivos de monitorização hemodinâmica (PVC, POAP) eletrólitos sé- } \\
\text { ricos normais, ausência de acidose ou alcalose metabólica. }\end{array}$ \\
\hline Glasgow escore & Avaliação do nível de consciência pela escala de coma de Glasgow $\geq 9$ \\
\hline Interrupção & $\begin{array}{l}\text { Uma vez avaliados os elementos acima a equipe deve definir ou não pela interrupção da ventilação me- } \\
\text { cânica. A resposta deverá ser sim ou não. }\end{array}$ \\
\hline Avaliação & $\begin{array}{l}\text { Caso a resposta seja negativa e o paciente permaneça em ventilação mecânica a equipe deverá definir a } \\
\text { periodicidade da nova avaliação. }\end{array}$ \\
\hline
\end{tabular}

$\mathrm{PaO}_{2}=$ pressão de oxigênio no sangue arterial; $\mathrm{FiO}_{2}=$ fração inspirada de oxigênio,

$\mathrm{PEEP}$ = pressão positiva no final da expiração, PVC = pressão venosa central;

POAP = pressão ocluída da artéria pulmonar ou pressão capilar pulmonar.

\section{CONSTRUINDO A ESTRATÉGIA}

O Consenso Brasileiro de Ventilação Mecânica definiu vários critérios para o desmame, baseado em evidências da literatura ${ }^{24}$. Sabe- se que na prática diária à beira do leito há a necessidade de simplificar a informação, planejar os objetivos a ser atingido, buscar a cooperação da equipe através de treinamento, programar o protocolo, reavaliar os resultados e, se necessário, reestruturar o protocolo. Os autores propõem um fluxograma de fácil interpretação e aplicação prática. (Figura 2).

\section{DISCUSSÃO}

Vincent ${ }^{21}$ propõe que, durante a ronda à beira do leito, a equipe utilize um método de memorização de uma sentença onde associa a cada letra os problemas que devem ser diariamente observados no paciente grave e que são passíveis de rápida detecção e correção. Através da frase FAST HUG (rápido abraço) são destacados aspectos clínicos e de boas práticas, como nutrição, analgesia e sedação, profilaxias da trombose venosa profunda, da pneumonia associada à ventilação mecânica e da úlcera de decúbito e o controle glicêmico. A elaboração de protocolos na atenção ao paciente grave visa promover a segurança e eficácia dos cuidados com o paciente. Também permite a realização de pesquisa e facilita o processo de

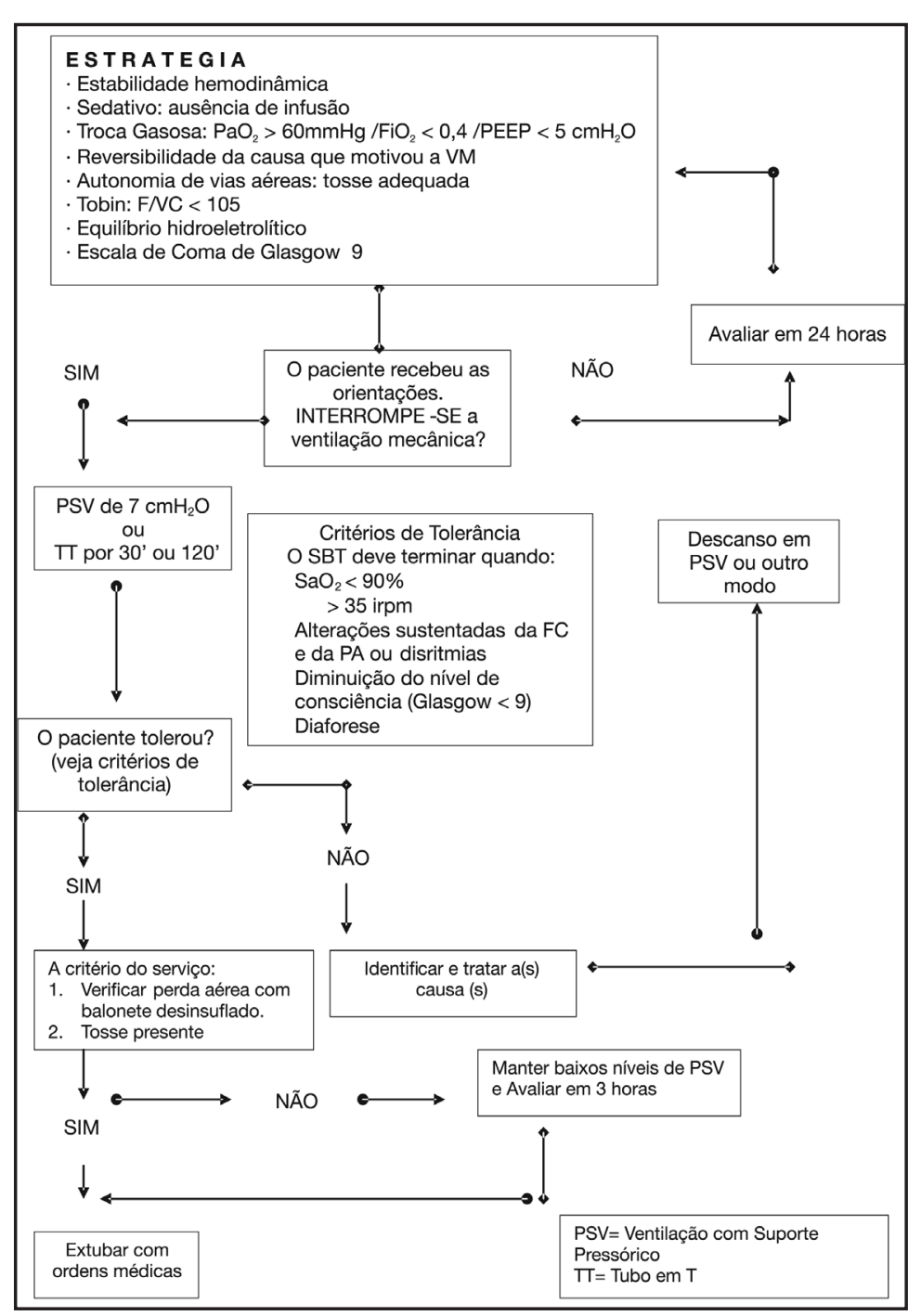

Figura 2 - Fluxograma de Desmame 
educação $0^{11,12,25}$. Protocolos para desmame conduzido pela equipe multiprofissional de saúde tem sido praticados por demonstrarem a capacidade em encurtar o tempo de desmame ${ }^{12}$. Wesley e col. ${ }^{3}$ comprovaram que o uso sistemático de intervenções produziu melhor resultado relacionado ao desmame do paciente.

A permanência do médico diarista nas UTI, como preconizado pela Associação de Medicina Intensiva Brasileira (AMIB) e publicado pelo Ministério da Saúde ${ }^{26}$ pode, em algumas situações, prescindir dos protocolos. As visitas do diarista e a equipe quando bem estruturadas resultam em melhor desfecho do paciente porque facilita, através da observação diária, a rápida detecção e pronta resolução de erros, como por exemplo, a manutenção do paciente em ventilação mecânica prolongada por sedação inapropriada ou por desnutrição ${ }^{27}$. Esta sistematização deve ser questionada, no mínimo uma vez ao dia ${ }^{21,25}$. Um estudo aleatório e controlado comprovou que em UTI fechadas com número de médicos especializados adequados ao número de leitos, o protocolo dirigido para o desmame não diminuiu o tempo de ventilação mecânica, as suas conseqüências fisiopatológicas e o custo hospitalar ${ }^{28}$. Por outro lado, protocolos parecem ser bastante úteis em unidades de terapia intensiva de hospitais com poucos leitos de UTI ou que se localizem em regiões mais distantes de grandes centros.

Uma alternativa aos protocolos seria a elaboração de checklists que possam ser facilmente realizados por qualquer profissional da equipe multidisciplinar ${ }^{21,25}$. A palavra ESTRATÉGIA foi sugerida pelos autores por se tratar do termo usado quando se quer atribuir uma tática especial a um problema baseado em evidência e experiência. Também pela flexibilidade que o termo admite, uma vez que a estratégia pode ser individualizada pela equipe baseando-se nas características daquela unidade de terapia intensiva. Por exemplo, uma unidade equipada com ventiladores que possam oferecer recursos tecnológicos mais avançados pode utilizá-lo como uma estratégia de desmame distinta de outra sem as mesmas soluções. Da mesma forma que, dependendo do perfil do paciente internado, como nos pacientes com tempo prolongado de ventilação por doenças neurológicas ou pulmonares crônicas, fosse importante acrescentar a avaliação da permeabilidade da via aérea através da avaliação feita com a desinsuflação do balonete antes de extubar.

Apesar de muitos estudos, sessões educacionais e envolvimento da equipe multidisciplinar, a aderência aos protocolos para desmame baseado em evidências tem sido de menos de $1 \%$ nas UTIS. Um estudo prospectivo instituindo um modelo de "Aceleração de Melhora Clínica" em desmame foi praticado em 129 pacientes consecutivos e 112 profissionais da equipe multidisciplinar e comparados os resultados do período anterior ao estudo. A freqüência das extubações sem sucesso diminuiu e a aderência da equipe aumentou. Não houve modificações em relação à pneumonia associada à ventilação mecânica, o tempo de ventilação mecânica e a percepção da prática mais segura ${ }^{29}$.

A permanência do paciente em assistência ventilatória deriva da incapacidade em manter adequada troca gasosa ou/e falência da bomba ventilatória. Estes fatos podem ser resultados de doença pulmonar (pneumonia, doença pulmonar crônica, asma e outros) ou extrapulmonar, mais notadamente do sistema nervoso central ou do sistema cardiovascular. Outras situações como desnutrição, distúrbios eletrolíticos, polineuropatia do paciente crítico reduzem a capacidade contrátil da musculatura ventilatória e devem ser identificadas. Nestas circunstâncias torna-se fundamental a busca da reversibilidade (total ou parcial) do processo que motivou a ventilação mecânica dentro da estratégia do desmame.

Aliada à evidência de melhora clínica deve-se ter oxigenação adequada e estabilidade hemodinâmica ${ }^{2,23,24}$. A percepção da melhora clínica, por se tratar de avaliação individual, traz consigo um caráter subjetivo. Este aspecto é minimizado quando a mesma equipe avalia o paciente diariamente ${ }^{11}$. Os protocolos para o manuseio de sedação e analgesia realizados por enfermeiras mostraram redução na duração da ventilação mecânica em dois dias $(p=0,008)$, redução do tempo de permanência em UTI em dois dias $(p<0,0001)$ e redução na incidência de traqueostomia no grupo de tratamento $(6 \% \times 13 \%, p=0,04)^{12}$. Por este motivo a importância em acrescentar a avaliação dos fármacos sedativos nas estratégias do desmame.

Os pacientes quando submetidos ao teste de interrupção da ventilação mecânica apresentam, temporariamente, piora da troca gasosa com aumento na diferença alvéolo-arterial de oxigênio e hipoxemia. A redução na pressão média de vias aéreas, com a retirada da ventilação à pressão positiva, pode ter impacto na oxigenação ${ }^{30}$. Os pacientes que evoluíram com piora clínica apresentaram esta alteração mais exacerbada do que aqueles que desmamavam com sucesso ${ }^{31}$. A piora da troca gasosa com hipoxemia pode ser explicada pelo aumento do curto-circuito arteriovenosos (shunt), provavelmente decorrente do rápido desenvolvimento 
de colapso alveolar com a retirada da pressão positiva. Também se deve suspeitar de falência cardíaca esquerda nos pacientes com história compatível, por alguns até considerados como grupo de risco para falência no desmame da ventilação mecânica ${ }^{32}$. Este fato pode ser explicado pelo aumento da pressão transmural do ventrículo esquerdo quando retirada à pressão positiva proporcionada pela ventilação mecânica que é de grande relevância nos pacientes com disfunção ventricular esquerda ${ }^{33,34}$.

Dos índices fisiológicos avaliados nos primeiros três minutos em ventilação espontânea, a freqüência respiratória, o volume corrente e a relação freqüência / volume-corrente (índice de respiração rápida superficial, no Brasil conhecido como índice de Tobin) apresentam boa acurácia para distinguir os pacientes que podem ser submetidos ao teste de 30 minutos a duas horas em ventilação espontânea ${ }^{22}$. Entretanto, pelo fato de estes testes estarem associadas a pequenas ou moderadas mudanças na probabilidade de sucesso e falha no desmame, não devem excluir pacientes considerados aptos à interrupção da ventilação mecânica mesmo quando seus resultados forem negativos ${ }^{1,2}$.

O tempo de desmame pode corresponder a até $50 \%$ do tempo total de ventilação mecânica, dependendo da doença que motivou a insuficiência respiratória ${ }^{14}$. A interrupção da ventilação mecânica, ou método do breve teste de respiração espontânea, é a técnica mais simples, estando entre as mais eficazes para o desmame. Faz-se permitindo que o paciente ventile espontaneamente através do tubo endotraqueal conectado a uma peça em forma de "T" com uma fonte enriquecida de oxigênio ou com ventilação à pressão de suporte (PSV) de $7 \mathrm{cmH}_{2} \mathrm{O}$ e deve ser tentado diariamente. Estes estudos mostraram que esta estratégia foi útil para selecionar os pacientes prontos para extubação ${ }^{2,13-18}$. Estes mesmos autores concluíram que o método ventilação mandatória intermitente sincronizada (SIMV) sem PSV está relacionado ao maior tempo em ventilação mecânica ${ }^{15,16}$. No entanto, apesar das evidências científicas, ainda se observam variações de métodos de desmame e resultados nas UTI em diversos países ${ }^{13}$. No Brasil esse fato também foi observado nas diferentes regiões do país ${ }^{20}$.

A interrupção da ventilação mecânica deve ser enfrentada como um momento distinto da extubação. Nos pacientes com prejuízo neurológico há estudos mostrando que apesar de alguns pacientes preencherem critérios para serem submetidos ao breve teste em ventilação espontânea houve um tempo médio de dois dias para que a ação fosse realizada. O motivo principal foi a preocupação com o baixo nível de consciência e a manutenção da autonomia ventilatória ${ }^{19}$. A avaliação do escore de coma Glasgow e da autonomia de vias aéreas pode ser uma ferramenta útil na estratégia dos pacientes neurológicos.

\section{CONCLUSÃO}

Sistematizar estratégias para o desmame nas UTI pode melhorar a qualidade do atendimento ao paciente em ventilação mecânica. Através da implantação de simples mecanismos de memorização que podem ser praticados durante a visita diária à beira do leito, é possível conseguir maior aderência da equipe e melhor evolução do paciente.

\section{REFERÊNCIAS}

01. Meade M, Guyatt $G$, Cook $D$ et al - Predicting success in weaning from mechanical ventilation. Chest, 2001;120:(Suppl6):400S-424S.

02. MacIntyre N, Cook DJ, Ely EW et al - Evidence-based guidelines for weaning and discontinuing ventilatory support: a collective task force facilitated by the American College of Chest Physicians; The American Association for Respiratory Care; and The American College of Critical Care Medicine. Chest, 2001;120:(Suppl6):S375- S39t.

03. Ely EW, Baker AM, Dunagan DP et al - Effect on the duration of mechanical ventilation of identifying patients capable of breathing spontaneously. N Engl J Med, 1996;335:1864-1869.

04. Fagon JY, Chastre J, Domart Y et al - Nosocomial pneumonia in patients receiving continuous mechanical ventilation: prospective analysis of 52 episodes with use of protected specimen brush and quantitative culture techniques. Am Rev Respir Dis, 1989;139:877-884.

05. Kollef MH, Shapiro SD, Silver $P$ et al - A randomized, controlled trial of protocol-directed versus physician-directed weaning from mechanical ventilation. Crit Care Med, 1997;25:567-574

06. Lemaire F,Teboul J, Cinotti $L$ et al - Acute left ventilatory dysfunction during unsuccessful weaning from mechanical ventilation. Anesthesiology, 1988; 69:1710179.

07. Pierson DJ - Complications associated with mechanical ventilation. Crit Care Clin, 1990;6:711-724.

08. Epstein SK, Nevins ML, Chunh J - Effect of unplanned extubation on outcome of mechanical ventilation. Am J Respir Crit Care Med, 2000; 161:1912-6.

09. Esteban A, Alia I, Gordo F et al - Extubation outcome after spontaneous breathing trials with T-tube or pressure support ventilation. The Spanish Lung Failure Collaborative Group. Am J Respir Crit Care Med, 1997;156:459-465.

10. Torres A, Gatell JM, Aznar E et al - Re-intubation increases the risks of nosocomial pneumonia in patients needing mechanical ventilation. Am J Respir Crit Care Med, 1995;152:137-141.

11. Ely EW, Meade MO, Haponik EF et al - Mechanical ventilator weaning protocols driven by nonphysician health-care professionals: evidence-based clinical practice guidelines. Chest, 2001;120:(Suppl6):S454S463.

12. Brook $A D$, Ahrens TS, Schaiff $R$ et al - Effect of a nursing implemented sedation protocol on the duration of mechanical ventilation. Crit Care Med, 1999;27:2609-2615

13. Esteban A, Anzueto A, Alia I et al - How is mechanical ventilation employed in the intensive care unit? An international utilization review. Am J Respir Crit Care Med, 2000;161:1450-1458.

14. Esteban A, Alia I Ibanez $\mathrm{J}$ et al - Modes of mechanical ventilation and weaning. A national survey of Spanish hospitals. The Spanish Lung Failure Collaborative Group. Chest, 1994;106:1188-1193 


\section{GOLDWASSER E DAVID}

15. Brochard L, Rauss A, Benito S et al - Comparison of three methods of gradual withdrawal from ventilatory support during weaning from mechanical ventilation. Am J Respir Crit Care Med, 1994;150:896-903.

16. Esteban A, Frutos F, Tobin MJ et al - A comparison of four methods of weaning patients from mechanical ventilation. Spanish Lung Failure Collaborative Group. N Engl J Med, 1995;332:345-350.

17. Esteban A, Alia I, Tobin MJ et al - Effect of spontaneous breathing trial duration on outcome of attempts to discontinue mechanical ventilation. Spanish Lung Failure Collaborative Group. Am J Respir Crit Care Med, 1999;159:512-518.

18. Perren A, Domenighetti G, Mauri S et al - Protocol-directed weaning from mechanical ventilation: clinical outcome in patients randomized for a 30-min or 120-min trial with pressure support ventilation. Intensive Care Med, 2002;28:1058-1063.

19. Namen AM, Wesley E, Tatter SB et al - Predictor of successful extubation in Neurosurgical Patients. Am J Respir Crit Care Med, 2001;163:658-664.

20. Damasceno MPCD - Estudo de Prevalência em Ventilação Mecânica no Brasil. Dissertação apresentada no programa de Pós Graduação em Clínica Médica-Terapia Intensiva da Faculdade de Medicina da Universidade Federal do Rio de Janeiro para a obtenção do título de mestre. Biblioteca da Faculdade de Medicina da UFRJ. 2005.

21. Vincent JL - Give your patient a fast hug (at least) once a day. Crit Care Med, 2005;33:1225-1229.

22. Yang KL, Tobin MJ - A prospective study of indexes predicting the outcome of trials of weaning from mechanical ventilation. $\mathrm{N}$ Engl $\mathrm{J}$ Med, 1991;324:1445-1450

23. Goldwasser R, Messeder O, Amaral JLG et al Desmame da Ventilação Mecânica. Relatório do II Consenso Brasileiro de Ventilação Mecânica; em: Carvalho CR - Ventilação Mecânica Vol I Clínicas Brasileiras de Medicina Intensiva, São Paulo, Atheneu, 2000.

24. Goldwasser R, Farias A, Saddy F et al - Desmame in III Consenso Brasileiro de Ventilação Mecânica. Disponível em http://www.amib.org.br
Acesso em 8 de Junho de 2006

25. Morris AH - Rational use of computerized protocols in the intensive care unit. Crit Care, 2001;5:249-254.

26. Estabelecimento de Critérios de Classificação para as unidades de tratamento intensivo. Portaria GM/MS número 1884 de 11 de Novembro de 1994, publicada em D.O. número 237, de 15 de Dezembro de 1994 Disponível em http://www.amib.org.br Acesso em 8 de Junho de 2006.

27. Dimick JB, Pronovost PJ, Heitmiller RF et al - Intensive Care Unit physician staff is associated with decreased length of stay, hospital cost, and complications after esophageal resection. Crit Care Med, 2001;29:753758.

28. Krishnan JA, Moore D, Robeson C et al - A prospective, controlled trial of a protocol-based strategy to discontinue mechanical ventilation. Am J Respir Crit Care Med, 2004;15;169:673-678.

29. Mc Lean S, Jensen MN, Schroeder DG et al - Improving adherence to a Mechanical Ventilation Weaning Protocol for Critically ill adults: outcomes after an implementation program. Am J Crit Care, 2006;15:299309

30. Dellinger RP - Weaning from Mechanical Ventilation, em: Vincent JL Yearbook of Intensive Care and Emergency Medicine. Springer-Verlag. 2006

31. Berry PR, Pontoppidan $\mathrm{H}$ - Oxygen consumption and bloog gas exchange during controlled ans spontaneous ventilation in patients with respiratory failure. Anethesiology, 1968;29:177.

32. Ferrer M, Valencia M, Nicolas JM et al - Early noninvasive ventilation averts extubation failure in patients at risk. Am J Respir Crit Care Med 2006;173:164-170.

33. David CM - Ventilação Mecânica: Repercussões hemodinâmicas, em David CM - Ventilação Mecânica. Da Fisiologia à Prática Clínica. Rio de Janeiro, Revinter, 2001.

34. Marini JJ, Culver BH, Butler J - Effect of positive end expiratory pressure on canine ventricular function. J Appl Physiol, 1981;51:1367-1374. 